\title{
Bubble Behavior and Heat Transfer in Quasi-Steady Pool Boiling in Microgravity
}

\author{
Jian-Fu Zhao · Jing Li • Na Yan · Shuang-Feng Wang
}

Received: 13 February 2009 / Accepted: 15 June 2009 / Published online: 7 July 2009

(C) Springer Science + Business Media B.V. 2009

\begin{abstract}
Pool boiling of degassed FC-72 on a plane plate heater has been studied experimentally in microgravity. A quasi-steady heating method is adopted, in which the heating voltage is controlled to increase exponentially with time. Compared with terrestrial experiments, bubble behaviors are very different, and have direct effect on heat transfer. Small, primary bubbles attached on the surface seem to be able to suppress the activation of the cavities in the neighborhoods, resulting in a slow increase of the wall temperature with the heat flux. For the high subcooling, the coalesced bubble has a smooth surface and a small size. It is difficult to cover the whole heater surface, resulting in a special region of gradual transitional boiling in which nucleate boiling and local dry area can co-exist. No turning point corresponding to the transition from nucleate boiling to film boiling can be observed. On the contrary, the surface oscillation of the coalesced bubble at low subcooling may cause more activated nucleate sites, and then the surface temperature may keep constant or even fall down with the increasing heat flux. Furthermore, an abrupt transition to film boiling can also be observed. It is shown that heat transfer coefficient and CHF increase with the subcooling or pressure in microgravity, as observed in normal gravity.
\end{abstract}

J.-F. Zhao $(\bowtie) \cdot$ J. Li · N. Yan · S.-F. Wang

National Microgravity Laboratory,

Institute of Mechanics,

Chinese Academy of Sciences (CAS),

15 Beisihuan Xilu, Beijing 100190, China

e-mail: jfzhao@imech.ac.cn
But the value of CHF is quite lower in microgravity, which may be only about one third of that at the similar pressure and subcooling in terrestrial condition.

Keywords Pool boiling - Bubble behavior . Heat transfer $\cdot$ Subcooling $\cdot$ CHF

\section{Introduction}

Pool boiling in microgravity has become an increasing significant subject for investigation, since many potential applications exist in space and in planetary neighbors due to its high efficiency in heat transfer. However, the investigation in microgravity suffers for unique and stringent constraints in terms of size, power and weight of experimental apparatuses, and of number and duration of the experiments. Thus, only a partial and in some aspects contradictory knowledge of microgravity boiling has been attained so far. On the progress in this field, several comprehensive reviews are available. For example, Straub (2001) issued a comprehensive review of his own activity on this field from the early 1980s to date, while Di Marco (2003), Kim (2003), Ohta (2003), and Wan and Zhao (2008) recently issued reviews of microgravity boiling researches in Europe, in US, in Japan, and in China, respectively. Therefore, only some researches dealing with pool boiling on plate heaters in microgravity, which are just the subject of the present study, are briefly summarized here.

Merte and coworkers (Lee et al. 1997; Merte et al. 1998) carried out a series of experiments in five different missions in a GAS facility on Space Shuttle in the period 1992-1996. Using the same hardware, pool boiling of R113 on a rectangular plate $\left(19 \times 38 \mathrm{~mm}^{2}\right)$ with 
various subcooling $(0.3 \sim 22.2 \mathrm{~K})$ at normal pressure conditions $(106.9 \sim 156.5 \mathrm{kPa})$ was investigated at heat fluxes up to $80 \mathrm{~kW} / \mathrm{m}^{2}$. The mechanism of steady boiling was described, which is in a substantial agreement with the observations of others. A large bubble resides a short distance from the heater and acts as a reservoir, engulfing small bubble forming on the heater. Due to balance of condensation at its cap and coalescence of new bubbles at the base, it maintains its size. Heat transfer was enhanced at low and intermediate heat flux, while degradation took place at higher heat flux. $\mathrm{CHF}$ was estimated to occur between 4 and $6 \mathrm{~W} / \mathrm{cm}^{2}$ except for the high subcooling case in microgravity, only about $1 / 3$ of that in normal gravity. Subcooling was found effective in enhancing heat transfer and in increasing the probability of having steady states. Migration of small bubbles from their location of origin towards the large bubble was observed for high subcooling. Bubble velocities of $2 \mathrm{~cm} / \mathrm{s}$ were common. A heat transfer enhancement of up to $40 \%$ was attributed to bubble migration. Marangoni convection was thought to cause this kind of motion.

Oka et al. (1995) performed several experiments on flat square plates with R113, water and n-pentane both in drop tower and in parabolic flight in the period 1989-1996.A mechanism quite analogous to that of Lee et al. (1997) was reported. Different bubble behaviors were observed for different liquids. For R113, bubbles tend to maintain a hemispherical shape with a large contact area with the heater. They remain attached to the surface at higher heat flux, and as a consequence, a situation of surface dryout is gradually attained with no sudden transition. Water bubbles exhibit "necking" and are readily detached from the heater. Latent heat of vaporization and surface tension are mainly responsible of the differences, and thus it appears that surface and fluid properties play an even greater role in microgravity than in normal gravity.

Pool boiling of ethanol on a flat disk of sapphire glass (50 mm dia.) was carried out by Ohta et al. (1999) in a Japanese sounding rocket TR-1A. The surface was indirectly heated by an ITO layer. Small Pt sensors were coated directly on the heated surface for local temperature and liquid film thickness measurements. The same boiling mechanism as described by Lee et al. (1997) was observed. Microlayers under the primary bubbles were occasionally replaced by dry patches. The measured thickness of the microlayer, variable in space and time, was between 10 and $100 \mu \mathrm{m}$. Enhancement of heat transfer was encountered. The authors reported that it is difficult to maintain steady pool boiling conditions over a long period in nearly saturated states due to the microlayer evaporation and extension of dry areas.
Straub and coworkers (Straub 2001) performed extensive experiments on microgravity pool boiling with various organic refrigerants and heater geometries from the early 1980s. They observed the same mechanism as described above for flat plates. A decrease of heat transfer coefficient was found. According to the wide experience of Straub, primary and secondary mechanisms were dominated for nucleate boiling. Primary mechanisms determine the nucleate boiling heat transfer, and they are to a large extent independent of gravity. The heat transfer is mainly caused by the evaporation in the so-called microwedge underneath the bubble. Secondary mechanisms provide the mass transport. This transport is caused mainly by buoyancy in normal gravity. In microgravity, buoyancy can largely be replaced by other secondary mechanisms, such as bubble coalescence processes, momentum of bubble growth and formation, and thermocapillary flow for subcooled states.

Kim et al. (2002) used a microheater array, consisting of $960.27-\mathrm{mm}$ square individual microheaters, to conduct pool boiling experiments of FC-72 in parabolic flight and sounding rocket. Their use of electronic feedback circuits to keep each of the heaters in the array at a constant temperature enables them to obtain local mapping of heat flux underneath growing bubbles. It was shown that the "nucleate boiling heat flux" collapsed onto a single curve, indicating that the small scale boiling was independent of subcooling and gravity level. It also suggests that $\mathrm{CHF}$ in microgravity simply results from the competition between increasing heat transfer from the satellite bubbles and the increase in the dry area under the primary bubble. Furthermore studies on the influences of the heater size and other factors have also been reported by the same group, e.g. Henry and Kim (2004).

The steady heating method, however, was usually employed in the studies mentioned above. The heat flux or surface temperature was adjusted step-by-step. For each step, the heating time lasted for a period long enough to obtain a steady state of boiling. It may, however, cause some difficulties in determining the boiling curves due to the large scatterance of measured data points. In the literature, quasi-steady heating method was also employed in the studying of boiling heat transfer in normal gravity to obtain continuous boiling curves which can be the same as those obtained in steady state case. For example, Johnson (1971) studied the quasi-steady condition by employed a very thin metallic ribbon for the heater in water boiling. The results demonstrated that transient nucleate boiling heat transfer for exponential heat inputs $q=q_{0} \exp \left(\tau / \tau_{0}\right)$, $\left(\tau_{0}\right)$ of less than $5 \mathrm{~ms}$, are reasonably well represented 
by steady state nucleate boiling correlation techniques. The value of $\mathrm{CHF}$ in a transient state case exceeds that in the corresponding steady state case. But the exceedance of CHF decreases with the increase of the period $\left(\tau_{0}\right)$, implying that the same CHF value can be reached if the period is long enough.

In the present study, we develop a device using a transient heating method with exponential heat inputs to conduct pool boiling experiment in microgravity aboard the Chinese recoverable satellite SJ-8 in order to obtain continuous, quasi-steady boiling curves in microgravity.

\section{Experimental Facility and Schedule}

The boiling chamber, as shown in Fig. 1, is fixed inside an air-proof container in which the pressure is initially about $100 \mathrm{kPa}$. A bellows connected with the chamber will allow the pressure in the chamber to be approximately constant during the boiling processes. Degassed FC-72 with a nominal volume of 1.71 was used as the working liquid. The liquid was degassed by periodically heating, reducing the pressure and cooling, allowing dissolved gases to diffuse out of the liquid. The process lasted for several days ensuring minimal gas concentrations. It has been argued that the presence of even minute amounts of dissolved gas can cause formation of thermocapillary convection around vapor bubbles in subcooled, microgravity environment (Straub 2001). Unfortunately, the final dissolved gas concentration in the liquid was not measured, and the extent to which the data was influenced by the dissolved gas is unknown.
The plane plate heater, as shown in Fig. 2, has an $\mathrm{Al}_{2} \mathrm{O}_{3}$ ceramic substrate with a size of $28 \times 20 \times 1 \mathrm{~mm}^{3}$ embedded in the PTFE base with a thickness of $25 \mathrm{~mm}$. An epoxy-bonded composite layer of mica sheets and asbestos is set between the ceram substrate and PTFE base to reduce the heat loss. The effective heating area with an area of $15 \times 15 \mathrm{~mm}^{2}$ is covered by a serpentine strip of multi-layer alloy film with a width of $300 \mu \mathrm{m}$ and a thickness about $10 \mu \mathrm{m}$. The space between the adjacent parallel strips is about $70 \mu \mathrm{m}$. The roughness of the heater surface is shown in Fig. 3. The multi-layer alloy film with a nominal resistance of $6 \Omega$ comprises several layers of metals $(\mathrm{Cr}, \mathrm{Cu}, \mathrm{Ni}, \mathrm{Au})$.

A transient heating method is adopted, in which the heating voltage is controlled as an exponential function with time, namely

$U=U_{0} \exp \left(\tau / \tau_{0}\right)$

where $\tau$ denotes the heating time, and the period $\tau_{0}$ determines the heating rate. In the space experiment aboard the Chinese recoverable satellite SJ- 8 and the control experiment on the ground before the space flight, the period is set for $\tau_{0}=80 \mathrm{~s}$ in order to make the heating process as a quasi-steady state, which was verified in the preliminary experiments on the ground. Furthermore, the period used in the present study is about $3 \sim 4$ order of magnitude larger than those in Johnson (1971), which guarantees the fulfillment of quasi-steady condition, though different structure of the heater and working fluid employed here.

In addition to as a heating element, the multi-layer alloy film also serves simultaneously as a resistance thermometer. The following correlation between the averaged temperature of the heater surface and the
Fig. 1 The boiling chamber and its accessories

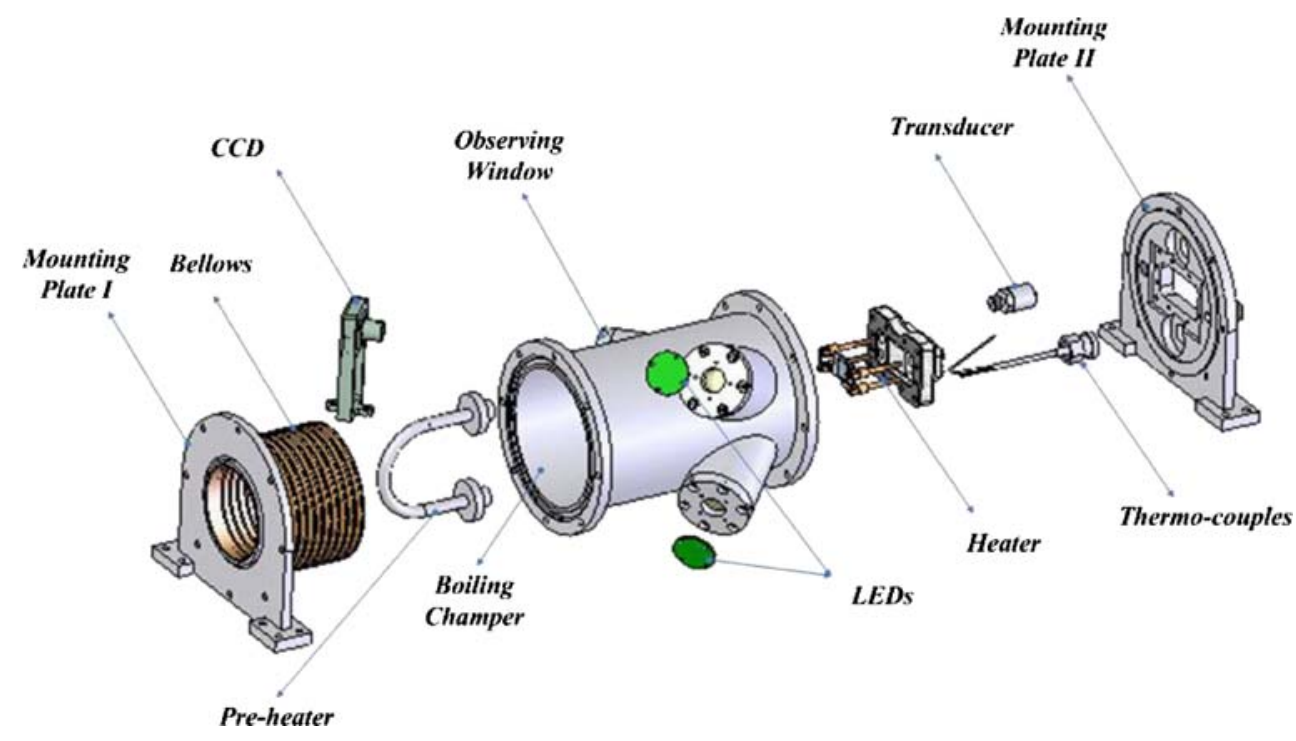




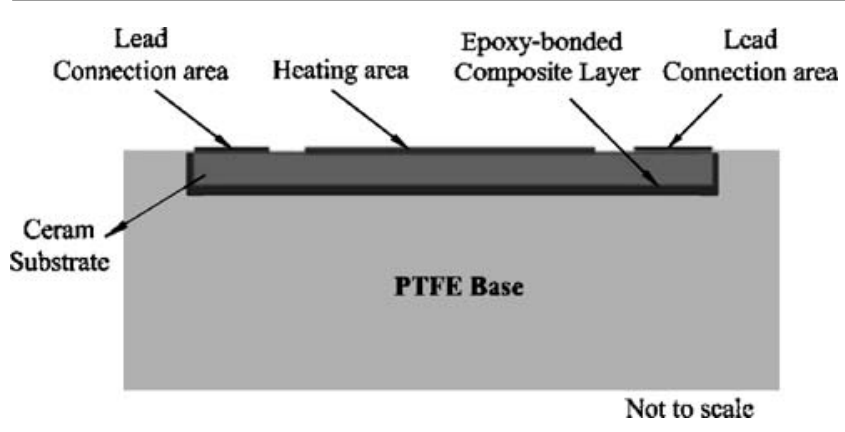

Fig. 2 The heater structure

resistance of the multi-layer alloy film was calibrated prior to the space flight

$t_{W}=-241.91087+48.77707 R_{W}$

The voltages across the heater and the electric current through the heater, which was measured by a Hall transducer, are sampled at a rate of $400 \mathrm{~Hz}$. A smaller storage rate of $100 \mathrm{~Hz}$, however, is used with 4-point averaging of the initial data due to the limit of data storage aboard the satellite. These data are used to determine the input heat flux with an uncertainty of $2.0 \%$ and the averaged temperature of the heater surface with an overall uncertainty of $2.0^{\circ} \mathrm{C}$.

In the following data reduction, the data of the averaged temperature of the heater surface are filtered to remove noise effects. The total heat flux input is transported into both the liquid and the $\mathrm{Al}_{2} \mathrm{O}_{3}$ ceramic substrate, while the heat loss to the PTFE base is neglected. The filtered temperature data can be used to compute the increase of the inner energy of the $\mathrm{Al}_{2} \mathrm{O}_{3}$ ceramic substrate using appropriate numerical computations. Subtracting the increase of the inner energy of the $\mathrm{Al}_{2} \mathrm{O}_{3}$ ceramic substrate from the total heat flux input provides the heat flux to the liquid and the transient mean heat transfer coefficient.

It ought to point out here some defects in the data reduction. After the incipience, the inner energy of the $\mathrm{Al}_{2} \mathrm{O}_{3}$ ceramic substrate decreases due to the dramatic drop of the heater temperature. It can be transported to both the liquid and the PTFE base. Since the heat loss to the PTFE base is neglected in the present data reduction, this part of heat flux is added entirely to the heat flux to the liquid, which may result in a slight mendacious increase of the heat flux to the liquid in the transitional nucleate boiling regime. On the other hand, the neglect of the heat loss to the PTFE base may also cause a slight underestimate of the heat flux to the liquid during the increase of the heater temperature.
Furthermore, at the beginning of heating, the heater temperature exhibits a jump from that in the bulk liquid due to the lagging of the data acquirement system, resulting in a great calculated increase of the inner energy of the $\mathrm{Al}_{2} \mathrm{O}_{3}$ ceramic substrate which can be even larger than the total heat flux input, and then a negative heat flux to the liquid. It is an untrue phenomenon. Its influence, however, will be neglectable as the increase of the heat flux.

The absolute pressure within the boiling chamber is measured using a pressure transducer with a range of $0 \sim 0.2 \mathrm{MPa}$ and an uncertainty of $0.25 \%$ FS (full scale). An auxiliary heater is used for adjusting the temperature of the bulk liquid from the ambient temperature to about the middle between the ambient and saturation temperature at the corresponding pressure. Two thermocouples with a range of $0 \sim 100^{\circ} \mathrm{C}$ and an uncertainty of $0.5^{\circ} \mathrm{C}$ are used to measure the bulk temperature of the fluid in the boiling chamber. The outputs for thermocouples and pressure transducer are sampled at rate of $1 \mathrm{~Hz}$. A CCD video camera, at the direction of $45^{\circ}$ with respect to the heater surface, is used to obtain images of the motion of vapor bubble or film around the heater, which is digitized and recorded at a speed of $25 \mathrm{fps}$ in MPEG. Four LEDs (lightemitting diode) are used to light the boiling chamber through two optical windows.

The space experiments, designed as two stages, were flown aboard the Chinese recoverable satellite SJ-8 in September 2006. Each stage includes several runs. Except the first run without pre-heating phase, every run consists of pre-heating, stabilizing and boiling phases, and lasts about $1 \mathrm{~h}$. The first stage is conducted in an ambient pressure condition with the initial pressure of about $100 \mathrm{kPa}$. After the first stage has been finished, a solenoid valve is opened to vent air from the container to the module of the satellite, and then the pressure inside the container was reduced to the same of that in the module of the satellite, namely $40 \sim 60 \mathrm{kPa}$.

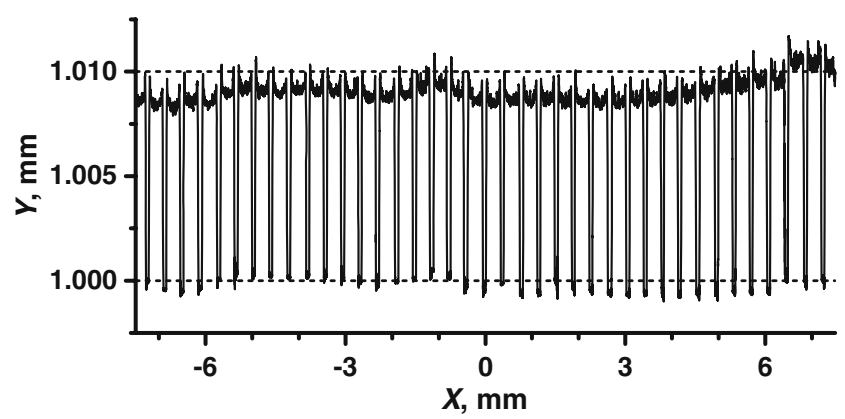

Fig. 3 The roughness of the heater surface 
Thus, in the second stage of the space experiment, the pressure in the boiling chamber is different from those in the first stage. One more stage is added in actual flight, which is also performed in the reduced pressure condition.

\section{Results and Discussions}

Before the space flight, several control experiments have been performed at the atmospheric pressure on the ground. Figure 4 shows the comparison of the results of the control experiments, in which the horizontal upward orientation was adopted for the heater surface, with those of steady boiling of FC-72 on plate heaters at similar conditions, such as pressure, subcooling and orientation (Rainey et al. 2003; Wei et al. 2005). It's found that the incipience temperature in the present transient boiling is consistent with that in the steady state (Wei et al. 2005), while the CHF value is also in agreement with those in the steady state. Comparing with the nucleate boiling curves in the steady state, the nucleate boiling curve in the present transient state locates in the similar range. Some differences exist, which may be caused by the differences in the heaters' size, structure, and surface condition in addition to the different heating methods.

There are 8 runs obtained in the space experiment. Unfortunately, video images have been obtained only in the five runs of the first stage of the space flight. The corresponding experimental conditions, the estimated values of $\mathrm{CHFs}$ and the corresponding superheats are

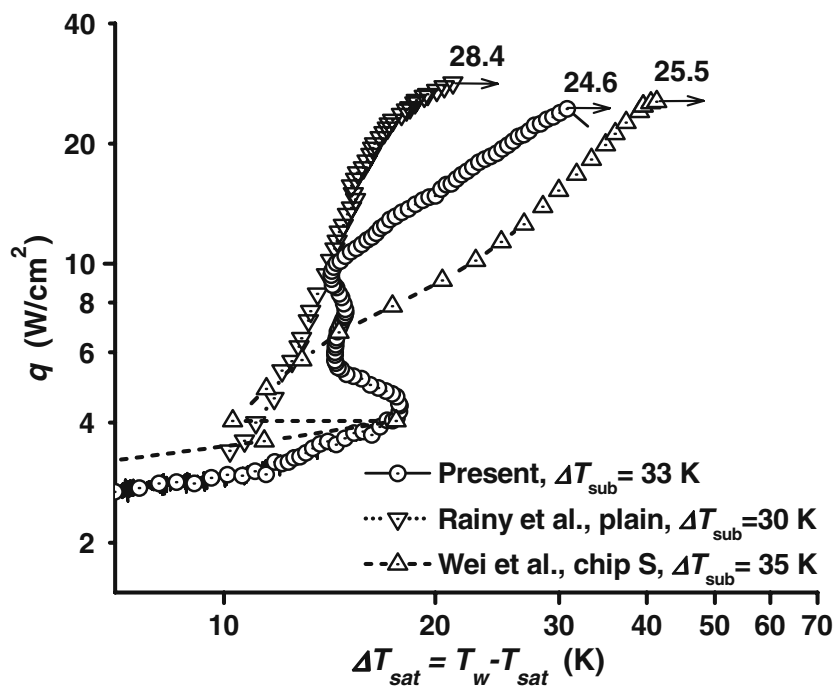

Fig. 4 Boiling curves in normal gravity all listed in Table 1. The estimating method for CHFs will be explained later. Figures 5, 6 and 7 show some typical processes of bubble generation and growth, the heating histories, and the corresponding boiling curves in the space experiments.

Because of the residual gravity which is estimated in the range of $10^{-3} \sim 10^{-5} g_{0}$, there could be a week single-phase natural convection before the incipience of boiling. In the five runs of the first stage, the first appearance of bubbles is observed at $21.89 \mathrm{~s}, 8.68 \mathrm{~s}$, $8.12 \mathrm{~s}, 4.54 \mathrm{~s}$, and $4.84 \mathrm{~s}$, respectively. Comparing with the first runs, the nucleate boiling occurred significantly earlier in the following runs.

In the first run I-1, a great amount of vapor appears abruptly and explosively at the incipience, as shown in Fig. 5a-A. Surface tension then compels the vapor to form several segregate bubbles. An obvious drop is observed in the history of the heater temperature (Fig. 5b and c), correspondingly. This drop of the heater temperature causes additional heat flux from the $\mathrm{Al}_{2} \mathrm{O}_{3}$ ceramic substrate to the liquid, and results in the maximum of the heat flux to the liquid in the transitional nucleate boiling regime despite of monotonous increasing of heating rate.

On the contrary, a gradual growth of the first bubble is observed in the following runs, e.g. the run I-2 as shown in Fig. 6a-A. The bubble growth process even appears an obvious standstill after its first appearance. Correspondingly, no over-shooting can be observed in the history of the heater temperature (Fig. 6b and c). Considering the experimental procedure, it may indicate that there could be residual micro-bubbles in cavities after the preceding runs. These micro-bubbles would make the cavities easier to be activated, and the boiling will thus be initiated at a lower wall superheat. Furthermore, bubbles attached on the surface seem to be able to suppress the activation of the cavities in the neighborhoods according to the detailed analyses of the video images. Thus, the temperature of the heater increases slowly with the increase of heat flux, and then the nucleate boiling curve has a considerably small slope in microgravity.

Since the influence of residual gravity on bubble behaviors is much weak in microgravity, it is hard for the bubbles to depart from the surface. Although the images were taken only from the sole direction of $45^{\circ}$ with respect to the heater surface, it can be observed that primary bubbles generate continually, slide on the surface, and coalesce with each other to form a larger coalesced bubble. Some primary bubbles can also generate under the coalesced bubble. The coalesced bubble also engulfs small bubbles around it. It can be 
Table 1 Space experimental conditions and the estimated $\mathrm{CHF}$ values

\begin{tabular}{lcllll}
\hline Run $^{\#}$ & $\begin{array}{l}\text { Pressure } \\
p(\mathrm{kPa})\end{array}$ & $\begin{array}{l}\text { Liquid } \\
\text { temperature } \\
t_{\mathrm{L}}\left({ }^{\circ} \mathrm{C}\right)\end{array}$ & $\begin{array}{l}\text { Subcooling } \\
\Delta T_{\text {sub }}(\mathrm{K})\end{array}$ & $\begin{array}{l}\text { CHF } q_{\text {CHF }} \\
\left(\mathrm{W} / \mathrm{cm}^{2}\right)\end{array}$ & $\begin{array}{l}\text { Corresponding } \\
\text { superheat } \\
\Delta T_{\text {sat }}(\mathrm{K})\end{array}$ \\
\hline $\mathrm{I}-1$ & 90.8 & 17.5 & 36.9 & $8.3 \sim 10.0$ & $28 \sim 66$ \\
$\mathrm{I}-2$ & 97.3 & 30.6 & 25.8 & $6.6 \sim 9.1$ & $34 \sim 76$ \\
$\mathrm{I}-3$ & 102.3 & 36.2 & 21.8 & $7.0 \sim 7.6$ & $40 \sim 56$ \\
$\mathrm{I}-4$ & 105.7 & 39.5 & 19.5 & $7.7 \sim 8.2$ & $20 \sim 29$ \\
$\mathrm{I}-5$ & 111.7 & 43.3 & 18.4 & $8.6 \sim 8.9$ & $11 \sim 17$ \\
II-1 & 57.2 & 16.7 & 24.5 & $5.7 \sim 6.9$ & $24 \sim 42$ \\
II-2 & 91.1 & 35.7 & 18.8 & $7.4 \sim 9.5$ & $26 \sim 55$ \\
III-1 & 65.5 & 17.5 & 27.5 & $6.3 \sim 6.6$ & $30 \sim 35$ \\
\hline
\end{tabular}

inferred that, as described above, a macro-layer may exist underneath the coalesced bubble, where primary bubbles are forming.

For the cases of higher subcooling, it can be observed that the coalesced bubble with a relative smooth surface oscillates near the center of the heater surface by interaction of the repulsion of the primary bubbles and the pulls of liquid flow surround it. Its size increases with the increase of the surface temperature, but it's very difficult to cover the whole surface. Higher is the subcooling, smaller and smoother the coalesced bubble at the same heating time. This fact is caused by the strong condensation near the top of the coalesced bubble, where the vapor connects directly with the subcooled liquid. Under the interaction between the surface tension, the coalesced bubble shrinks to an elliptical sphere. Thus, the bottom of the coalesced bubble may dry out partly at high heat flux, while the other place, particularly in the corners, of the heater surface can be still in the region of nucleate boiling. With the developing of the size of the coalesced bubble and the area of local dry area, the boiling pattern will gradually change to film boiling, as described by Oka et al. (1995). This kind of transitional boiling leads to a much smooth increase of the averaged temperature of the heater surface and no turning point appearing in the corresponding boiling curves. However, the trend of the increasing heater temperature with the heating time
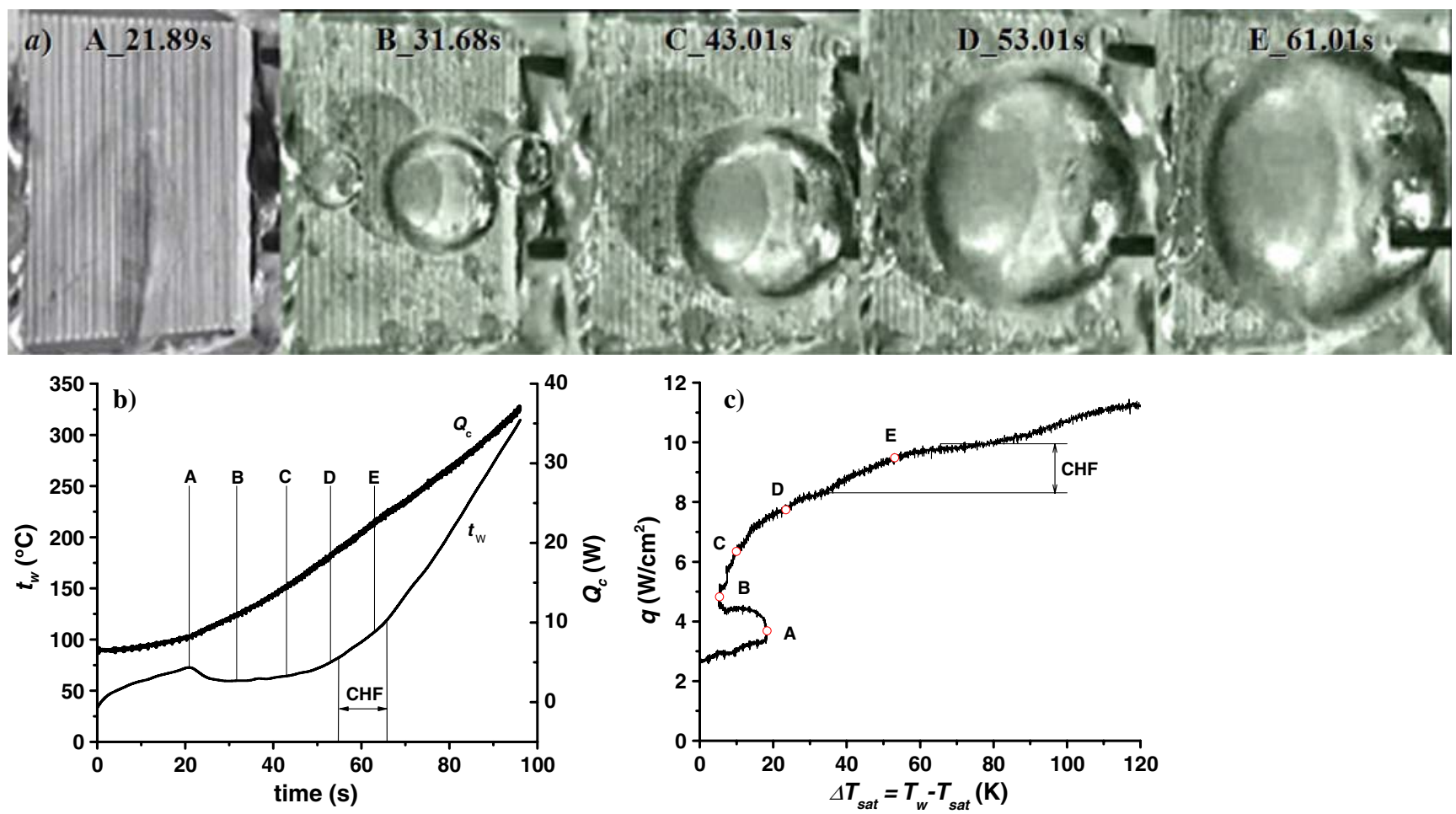

Fig. 5 Bubble behaviors (a), heating history (b), and boiling curve (c) in the run I-1 

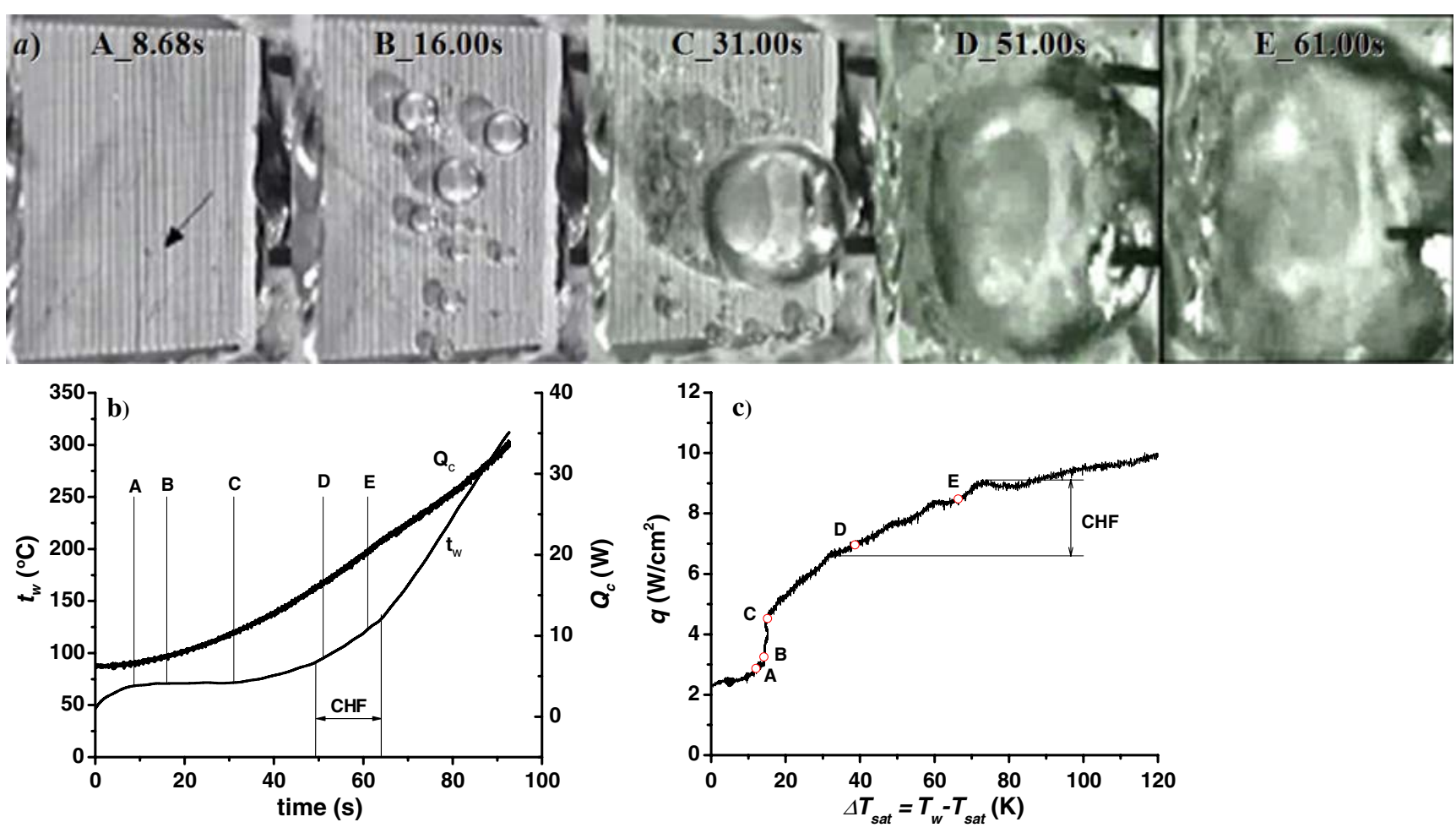

Fig. 6 Bubble behaviors (a), heating history (b), and boiling curve (c) in the run I-2

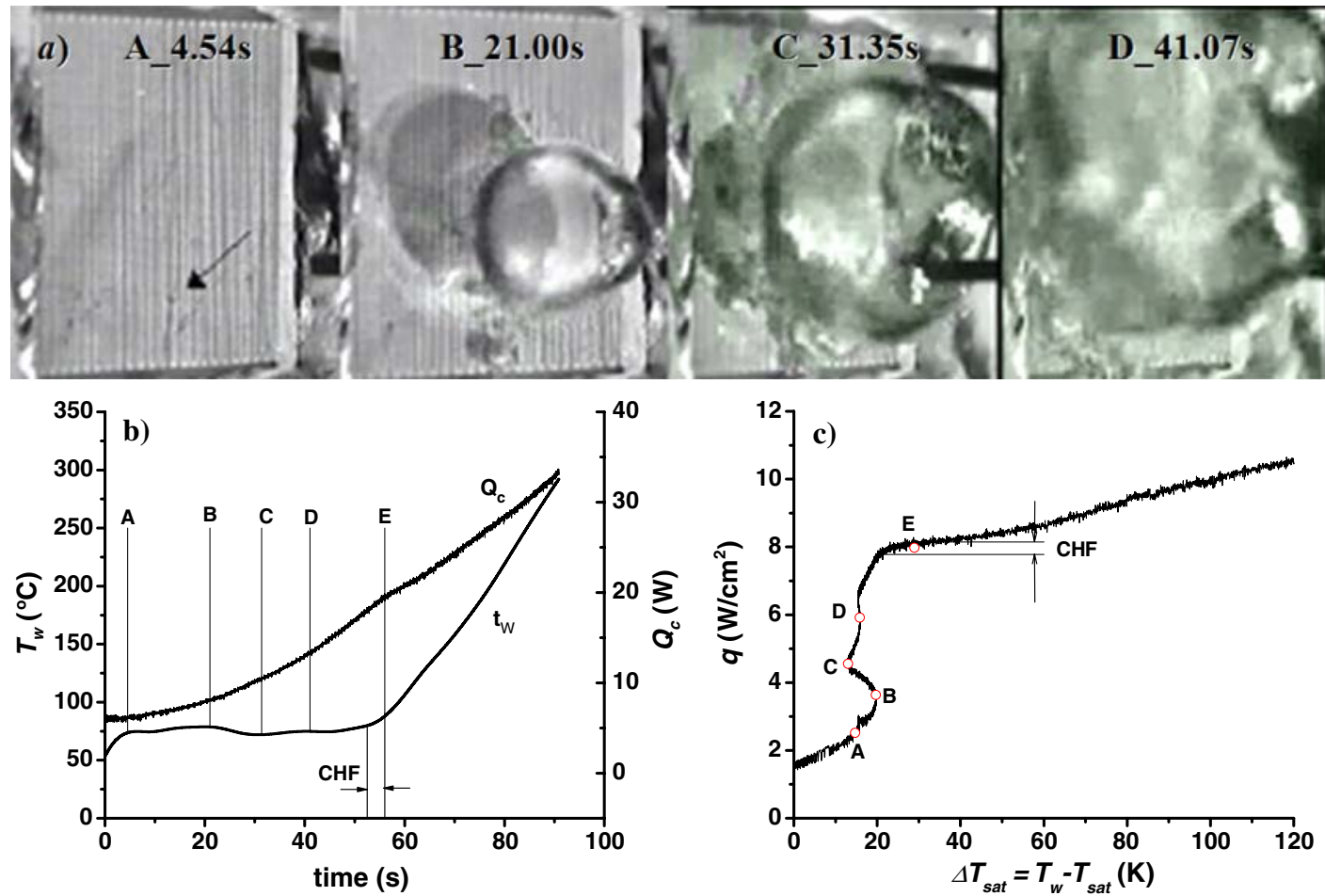

Fig. 7 Bubble behaviors (a), heating history (b), and boiling curve (c) in the run I-4 


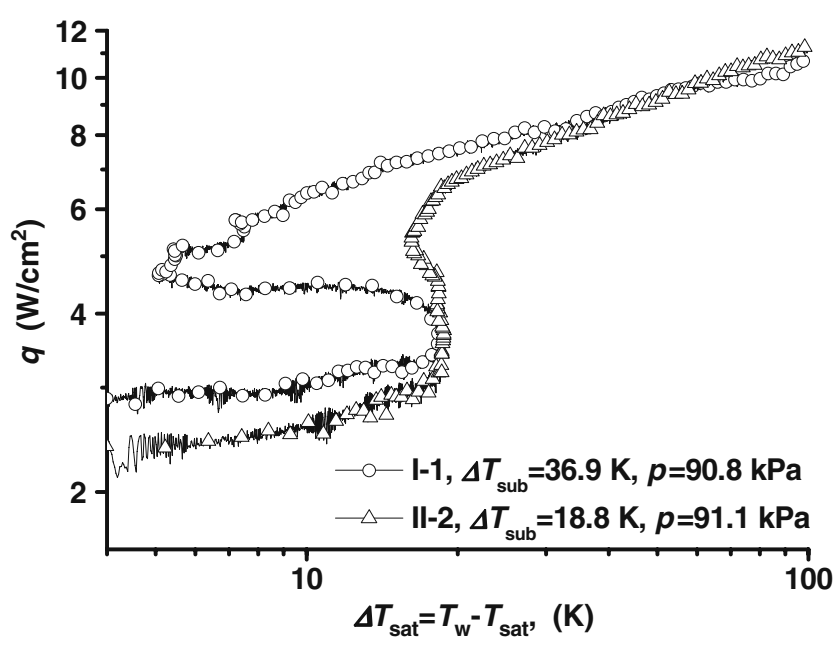

Fig. 8 Subcooling influence on boiling curves

can provide some information of $\mathrm{CHF}$, namely a rapid increase may indicate the beginning of the transitional boiling, while a constant slope of the temperature curve may suggest the complete transition to film boiling. The corresponding ranges are marked in Figs. 5, 6 and 7, and the values of $\mathrm{CHF}$ and corresponding superheats are estimated for all the space experimental runs and also listed in Table 1.

The bubble behavior and the characteristic of the boiling curves at lower subcooling, such as in the runs I-4, are different from others at higher subcooling. In these runs, bubbles are difficult to condense due to the relatively lower subcooling. The size of the coalesced bubble increases quickly, and a strong oscillation caused by continuous coalescences appears on their

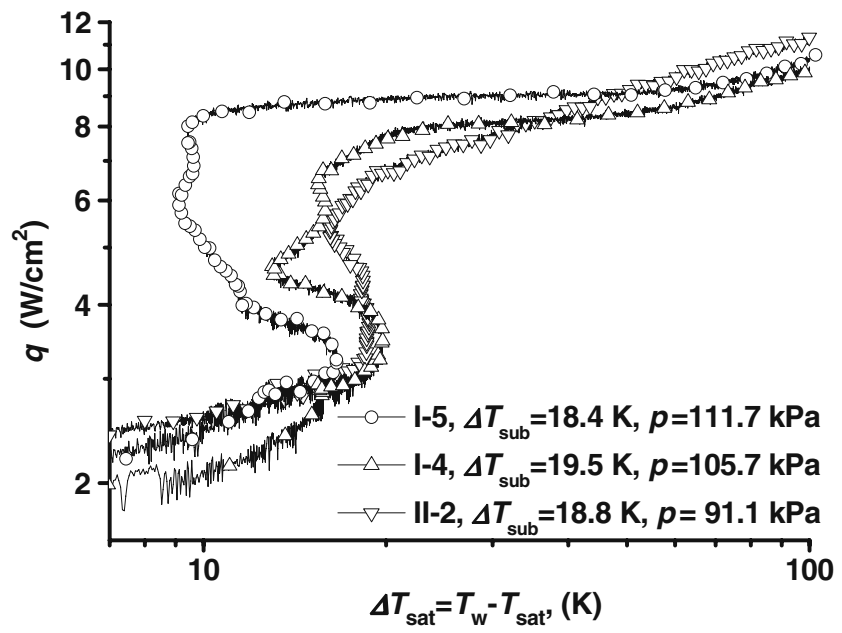

Fig. 9 Pressure influence on boiling curves

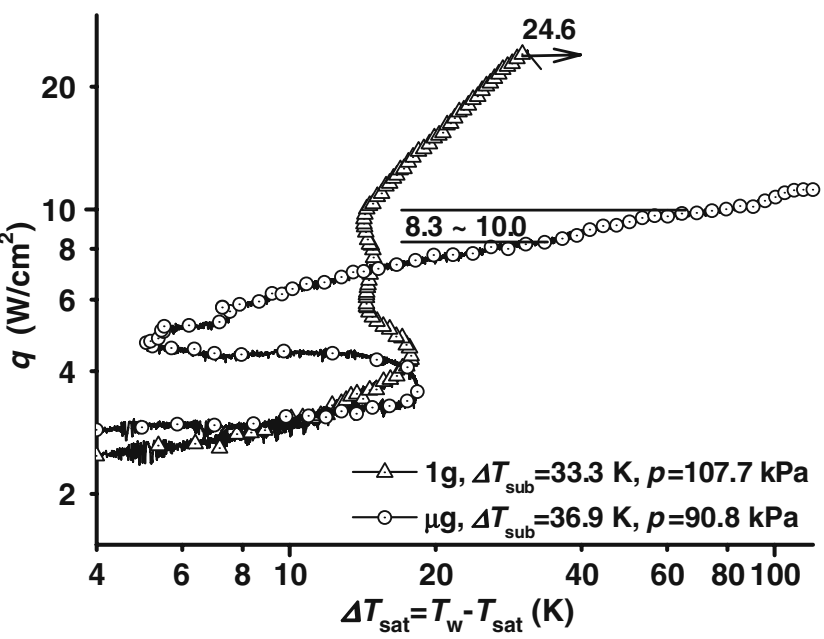

Fig. 10 Comparison of boiling curves in different gravity

surface. Furthermore, higher is pressure, stronger the surface oscillation because the surface tension on the coalesced bubble decreases with the increase of the saturation temperature and the corresponding pressure. Thus, local dry spots underneath the coalesced bubble can not develop steadily. They may be re-wetted by the surrounding liquid, and nucleate boiling will remain on the heater surface. Under the action of the strong oscillation of the coalesced bubble, even more nucleate sites will be activated. Thus, unless the abrupt transition to film boiling, the heat flux will keep increasing although the surface temperature rises slowly or even fall down. In these cases, turning points corresponding to $\mathrm{CHF}$ will be observed in the boiling curves. The ranges determined according to the same method as in the other runs are also marked in Fig. 7, which are much narrower.

The influences of subcooling and pressure on boiling curves in microgravity are shown in Figs. 8 and 9, respectively. At the same pressure, heat transfer will be deteriorated with the decrease of subcooling, but be enhanced with the increase of pressure at the same subcooling.

The estimated values of CHF in microgravity based on the analysis of the trend of the increasing heater temperature with the heating time, which are listed in Table 1, will also increase with the subcooling at the same pressure, and increase with pressure at the same subcooling. These trends are similar with those observed in normal gravity, while the value of $\mathrm{CHF}$ in microgravity is only about one third of that at the similar pressure and subcooling in terrestrial condition. Unfortunately, the pressure and temperature of the liquid cannot be isolated completely because of the 
passive control of the pressure inside the boiling chamber used in the present study. Thus, some crossinfluences of pressure and subcooling on CHF exist.

In Fig. 10, boiling curves in different gravity conditions are compared with each other at the similar pressure and subcooling conditions. Generally, boiling heat transfer in microgravity is deteriorated comparing that in normal gravity, particularly at high superheats or heat fluxes. Much obvious enhancement, however, can be observed just beyond the incipience, which is consistent with those in steady state pool boiling experiments, such as reported by Lee et al. (1997).

\section{Conclusions}

In the present paper, the bubble behaviors and heat transfer in quasi-steady pool boiling of FC-72 on a plane plate heater have been studied experimentally in microgravity aboard the Chinese recoverable satellite SJ-8.

Compared with terrestrial experiments, bubble behaviors is very different, and have a direct effect on the characteristic of heat transfer. Small, primary bubbles attached on the surface seem to be able to suppress the activation of the cavities in the neighborhoods, resulting in a slow increase of the wall temperature with the heat flux. For the high subcooling, the coalesced bubble has a smooth surface and a small size. It is difficult to cover the whole heater surface, resulting in a special region of gradual transitional boiling in which nucleate boiling and local dry area can co-exist. No turning point corresponding to the transition from nucleate boiling to film boiling can be observed. On the contrary, the surface oscillation of the coalesced bubble at low subcooling may cause more activated nucleate sites, and then the surface temperature may keep constant or even fall down with the increasing heat flux. Furthermore, an abrupt transition to film boiling can also be observed, and then an obvious turning point corresponding to $\mathrm{CHF}$ appears on the boiling curve.

It is shown that heat transfer coefficient and CHF increase with the subcooling or pressure in microgravity, as observed in normal gravity. But the value of CHF is quite lower in microgravity, which may be only about one third of that at the similar pressure and subcooling in terrestrial condition.
Acknowledgements The present study is supported financially by the Chinese Academy of Sciences under the grant of KACX2SW-02-03, and the National Natural Science Foundation of China under the grant of 10432060. The authors thank Prof. H. Ohta of the Kyushu University, Japan for his valuable advice in the preparation of the project and for the fruitful discussions. We also really appreciate the reviewers' comments, which help us to avoid a severe mistake in the data reduction and to improve greatly the quality of the paper.

\section{References}

Di Marco, P.: Review of reduced gravity boiling heat transfer: European research. J. Jpn. Microgravity Appl. 20, 252-263 (2003)

Henry, C.D., Kim, J.: A study of the effects of heater size, subcooling, and gravity level on pool boiling heat transfer. Int. J. Heat Fluid Flow 25, 262-273 (2004)

Johnson, H.A.: Transient boiling heat transfer to water. Int. J. Heat Mass Transfer 14, 67-82 (1971)

Kim, J.: Review of reduced gravity boiling heat transfer: US research. J. Jpn. Microgravity Appl. 20, 264-271 (2003)

Kim, J., Benton, J.F., Wisniewski, D.: Pool boiling heat transfer on small heaters: effect of gravity and subcooling. Int. J. Heat Mass Transfer 45, 3919-3932 (2002)

Lee, H.S., Merte, H., Chiaramonte, F.: Pool boiling curve in microgravity. J. Thermophys. Heat Transf. 11, 216-222 (1997)

Merte H., Lee H.S., Keller K.B.: Dryout and rewetting in the pool boiling experiment flown on STS-72 (PBE-IIB) and STS-77 (PBE-IIA). NASA/CR-1998-207410 (1998)

Ohta, H.: Review of reduced gravity boiling heat transfer: Japanese research. J. Jpn. Microgravity Appl. 20, 272-285 (2003)

Ohta, H., Kawasaki, K., Azuma, H., Yoda, S., Nakamura, T.: On the heat transfer mechanisms in microgravity nucleate boiling. Adv. Space Res. 24(10), 1325-1330 (1999)

Oka, T., Abe, Y., Mori, Y.H., Nagashima, A.: Pool boiling of n-pentane, CFC-113 and water under reduced gravity: parabolic flight experiments with a transparent heater. J. Heat Transfer, Trans. ASME 117, 408-417 (1995)

Rainey, K.N., You, S.M., Lee, S.: Effect of pressure, subcooling, and dissolved gas on pool boiling heat transfer from microporous surfaces in FC-72. J. Heat Transfer, Trans. ASME 125, 75-83 (2003)

Straub, J.: Boiling heat transfer and bubble dynamics in microgravity. Adv. Heat Transf. 35, 57-172 (2001)

Wan, S.X., Zhao, J.F.: Pool boiling in microgravity: recent results and perspective for the project DEPA-SJ10. Microgravity Sci. Technol. 20, 219-224 (2008)

Wei, J.J., Guo, L.J., Honda, H.: Experimental study of boiling phenomena and heat transfer performances of FC-72 over micro-pin-finned silicon chips. Heat Mass Transf. 41(8), 744755 (2005) 